J. Korean Math. Soc. 52 (2015), No. 1, pp. 97-111

http://dx.doi.org/10.4134/JKMS.2015.52.1.097

\title{
ON WEAKLY 2-ABSORBING PRIMARY IDEALS OF COMMUTATIVE RINGS
}

\author{
Ayman Badawi, Unsal TekiR, and Ece Yetkin
}

\begin{abstract}
Let $R$ be a commutative ring with $1 \neq 0$. In this paper, we introduce the concept of weakly 2 -absorbing primary ideal which is a generalization of weakly 2-absorbing ideal. A proper ideal $I$ of $R$ is called a weakly 2-absorbing primary ideal of $R$ if whenever $a, b, c \in R$ and $0 \neq a b c \in I$, then $a b \in I$ or $a c \in \sqrt{I}$ or $b c \in \sqrt{I}$. A number of results concerning weakly 2 -absorbing primary ideals and examples of weakly 2-absorbing primary ideals are given.
\end{abstract}

\section{Introduction}

We assume throughout this paper that all rings are commutative with $1 \neq 0$. Let $R$ be a commutative ring. An ideal $I$ of $R$ is said to be proper if $I \neq R$. Let $I$ be a proper ideal of $R$. Then $\sqrt{I}=\left\{r \in R: r^{k} \in I\right.$ for some $\left.k \in \mathbb{N}\right\}$ denotes the radical ideal of $R$ and $Z_{I}(R)=\{r \in R \mid r s \in I$ for some $s \in R \backslash I\}$. Note that $\sqrt{0}$ is the set (ideal) of all nilpotent elements of $R$. The concept of 2-absorbing ideal, which is a generalization of prime ideal, was introduced by Badawi in [5] and studied in [3], [12], and [8]. Various generalizations of prime ideals are also studied in [1] and [9].

Recall that a proper ideal $I$ of $R$ is called a 2-absorbing ideal of $R$ if whenever $a, b, c \in R$ and $a b c \in I$, then $a b \in I$ or $a c \in I$ or $b c \in I$. Recently (see [7]), the concept of 2-absorbing ideal is extended to the context of 2-absorbing primary ideal which is a generalization of primary ideal. Recall from [7] that a proper ideal of $R$ is said to be a 2-absorbing primary ideal of $R$ if whenever $a, b, c \in R$ with $a b c \in I$, then $a b \in I$ or $a c \in \sqrt{I}$ or $b c \in \sqrt{I}$. Recall from [2] ([4]) that a proper ideal $I$ of $R$ is called a weakly prime ideal (weakly primary ideal) if whenever $0 \neq a b \in I$, then $a \in I$ or $b \in I(a \in I$ or $b \in \sqrt{I})$. The concept of weakly prime ideal was extended to the context of weakly 2 -absorbing ideal. Recall from [6] that a proper ideal $I$ of $R$ is said to be a weakly 2-absorbing

Received March 18, 2014; Revised July 2, 2014.

2010 Mathematics Subject Classification. Primary 13A15; Secondary 13F05, 13G05.

Key words and phrases. primary ideal, weakly primary ideal, prime ideal, weakly prime ideal, 2-absorbing ideal, n-absorbing ideal, weakly 2-absorbing ideal, 2-absorbing primary ideal, weakly 2-absorbing primary ideal. 
ideal of $R$ if whenever $0 \neq a b c \in I$, then $a b \in I$ or $a c \in I$ or $b c \in I$. In this paper, we extend the concept of weakly 2-absorbing ideal to the context of weakly 2-absorbing primary ideal. A proper ideal $I$ of $R$ is said to be a weakly 2-absorbing primary ideal of $R$ if whenever $a, b, c \in R$ with $0 \neq a b c \in I$ implies $a b \in I$ or $a c \in \sqrt{I}$ or $b c \in \sqrt{I}$.

Note that every 2 -absorbing primary ideal is clearly a weakly 2 -absorbing primary ideal. However, the converse is not true. For example, 0 is always a weakly 2-absorbing primary ideal of $R$, but it is not always a 2-absorbing primary ideal.

Among many results in this paper, it is shown (Example 2.6) that the radical of a weakly 2-absorbing primary ideal of a $\operatorname{ring} R$ need not be a weakly 2 absorbing ideal of $R$. It is shown (Theorem 2.7) that if $I$ is a proper ideal of $R$ such that $\sqrt{I}$ is a weakly prime ideal of $R$, then $I$ is a weakly 2-absorbing primary ideal of $R$. It is shown (Theorem 2.10) that if $I$ is a weakly 2-absorbing primary ideal of $R$ that is not 2-absorbing primary, then $I^{3}=0$. It is shown (Example 2.11) that if $I^{3}=0$ for some proper ideal $I$ of $R$, then $I$ need not be a weakly 2 -absorbing primary ideal of $R$. It is shown (Theorem 2.14) that if $\sqrt{0}$ is prime and $I$ is a proper ideal of $R$, then $I$ is a weakly 2-absorbing primary ideal of $R$ if and only if $I$ is a 2-absorbing primary ideal. If $R=R_{1} \times \cdots \times R_{n}$, then a complete characterization of the nonzero weakly 2 -absorbing primary ideals of $R$ is determined (Theorem 2.21-Theorem 2.24). It is shown (Theorem 2.25) that every proper ideal of $R=R_{1} \times R_{2} \times R_{3}$ is a weakly 2-absorbing primary ideal of $R$ if and only if $R_{1}, R_{2}$, and $R_{3}$ are fields. It is shown (Theorem 2.26) that if every proper ideal of $R$ is weakly 2-absorbing primary, then $R$ has at most three incomparable (under inclusion) prime ideals (and hence at most three distinct maximal ideals). It is shown (Theorem 2.30) that if $I$ is a weakly 2-absorbing primary ideal of $R$ and $0 \neq I_{1} I_{2} I_{3} \subseteq I$ for some ideals $I_{1}, I_{2}, I_{3}$ of $R$ such that $I$ is free triple-zero with respect to $I_{1} I_{2} I_{3}$, then $I_{1} I_{2} \subseteq I$ or $I_{1} I_{3} \subseteq \sqrt{I}$ or $I_{2} I_{3} \subseteq \sqrt{I}$. In the last section, we give alternative proofs to some results in [2].

\section{Weakly 2-absorbing primary ideals}

Definition 2.1. A proper ideal $I$ of $R$ is called a weakly 2-absorbing primary ideal of $R$ if whenever $a, b, c \in R$ and $0 \neq a b c \in I$, then $a b \in I$ or $a c \in \sqrt{I}$ or $b c \in \sqrt{I}$.

Definition 2.2. Let $I$ be a weakly 2-absorbing primary ideal of $R$. We say $(a, b, c)$ is a triple-zero of $I$ if $a b c=0, a b \notin I, b c \notin \sqrt{I}$, and $a c \notin \sqrt{I}$.

Note that if $I$ is a weakly 2 -absorbing primary ideal of $R$ that is not 2absorbing primary ideal, then there exists a triple-zero $(a, b, c)$ of $I$ for some $a, b, c \in R$.

We start with the following result. We omit the proof since it is clear by definitions. 
Theorem 2.3. Let $I$ be a proper ideal of $R$. Then

(1) If $I$ is a weakly prime ideal, then $I$ is a weakly 2-absorbing primary ideal.

(2) If I is a weakly 2-absorbing ideal, then I is a weakly 2-absorbing primary ideal.

(3) If I is a weakly primary ideal, then I is a weakly 2-absorbing primary ideal.

(4) If I is a 2-absorbing ideal, then I is a weakly 2-absorbing primary ideal.

(5) If $I$ is a 2-absorbing primary ideal, then $I$ is a weakly 2-absorbing primary ideal.

Recall that a ring $R$ is called quasilocal if it has exactly one maximal ideal. The proof of the following result is clear, and hence we omit the proof.

Theorem 2.4. Let $R$ be a quasilocal ring with maximal ideal $\sqrt{0}$. Then every proper ideal of $R$ is a weakly 2-absorbing primary ideal of $R$.

Theorem 2.5. Let $I$ be a proper ideal of $R$. Then $\sqrt{I}$ is a weakly 2-absorbing ideal of $R$ if and only if $\sqrt{I}$ is a weakly 2-absorbing primary ideal of $R$.

Proof. Since $\sqrt{\sqrt{I}}=\sqrt{I}$, the proof is completed.

If $I$ is a 2-absorbing primary ideal of $R$, then $\sqrt{I}$ is a 2-absorbing ideal of $R$ by [7, Theorem 2.2]. However, if $I$ is a weakly 2-absorbing primary ideal, then $\sqrt{I}$ need not be a weakly 2 -absorbing ideal of $R$. We have the following example.

Example 2.6. Let $A=\mathbb{Z}_{2}[X, Y, W]$ and $I=X^{2} Y^{2} W^{2} A$ be an ideal of $A$. Let $R=A / I$. Then $I / I$ is the zero ideal of $R$, and hence 0 is a weakly 2-absorbing primary ideal of $R$. We show that $\sqrt{0}$ (in $R$ ) $=X Y W A / I$ is not a weakly 2-absorbing ideal of $R$. For in the ring $R$, we have $0 \neq X Y W+I \in \sqrt{0}$, but $X Y+I \notin \sqrt{0}, X W+I \notin \sqrt{0}$, and $Y W+I \notin \sqrt{0}$. Thus $\sqrt{0}$ (in $R$ ) is not a weakly 2-absorbing ideal of $R$.

Let $I$ be a proper ideal of $R$. Since $\sqrt{I}=\sqrt{\sqrt{I}}$, it is clear that $\sqrt{I}$ is a weakly prime ideal of $R$ if and only if $\sqrt{I}$ is a weakly primary ideal of $R$. Hence we have the following result.

Theorem 2.7. Let $I$ be a proper ideal of $R$ such that $\sqrt{I}$ is a weakly prime (weakly primary) ideal of $R$. Then $I$ is a weakly 2-absorbing primary ideal of $R$.

Proof. Suppose that $0 \neq a b c \in I$ for some $a, b, c \in R$ and $a b \notin I$. Suppose that $a b \notin \sqrt{I}$. Since $\sqrt{I}$ is a weakly prime ideal of $R$, we have $c \in \sqrt{I}$, and thus $a c \in \sqrt{I}$. Suppose that $a b \in \sqrt{I}$. Since $0 \neq a b c \in I$ and $a b \in \sqrt{I}$, we have $0 \neq a b \in \sqrt{I}$. Since $\sqrt{I}$ is a weakly prime ideal of $R$ and $0 \neq a b \in \sqrt{I}$, we have $a \in \sqrt{I}$ or $b \in \sqrt{I}$. Thus $a c \in \sqrt{I}$ or $b c \in \sqrt{I}$. Thus $I$ is a weakly 2-absorbing primary ideal of $R$. 
Theorem 2.8. Let $I$ be a weakly primary ideal of $R$ that is not primary and $J$ be an ideal of $R$ such that $J \subseteq I$. Then $J$ is a weakly 2-absorbing primary ideal of $R$. In particular, if $L$ is an ideal of $R$, then $A=I \cap L$ and $B=I L$ are weakly 2-absorbing primary ideals of $R$.

Proof. Since $I$ is a weakly primary ideal of $R$ that is not primary, $\sqrt{I}=\sqrt{0}$ by $[4$, Theorem 2.2]. Hence $\sqrt{J}=\sqrt{I}=\sqrt{0}$. Let $0 \neq a b c \in J$ for some $a, b, c \in R$ and suppose that $a b \notin J$. Since $J \subseteq I$, we have $0 \neq a b c \in I$. We consider two cases. Case one: Suppose that $a b \notin I$. Since $I$ is weakly primary and $a b \notin I$, we have $c \in \sqrt{J}=\sqrt{I}=\sqrt{0}$. Thus $a c \in \sqrt{J}$. Case two: Suppose that $a b \in I$. Since $0 \neq a b c \in I$, we have $0 \neq a b \in I$. Since $I$ is a weakly primary ideal of $R$, we have $a \in I \subseteq \sqrt{0}$ or $b \in \sqrt{0}$. Thus $a c \in \sqrt{J}$ or $b c \in \sqrt{J}$. Thus $J$ is a weakly 2 -absorbing primary ideal of $R$. The proof of the "in particular statement" is clear since $A, B \subseteq I$.

Theorem 2.9. Let $I$ be a weakly 2-absorbing primary ideal of $R$ and suppose that $(a, b, c)$ is a triple-zero of I for some $a, b, c \in R$. Then

(1) $a b I=b c I=a c I=0$.

(2) $a I^{2}=b I^{2}=c I^{2}=0$.

Proof. (1) Suppose that $a b I \neq 0$. Then there exists $i \in I$ such that $a b i \neq 0$. Hence $a b(c+i) \neq 0$. Since $a b \notin I$ and $I$ is weakly 2-absorbing primary, we have $a(c+i) \in \sqrt{I}$ or $b(c+i) \in \sqrt{I}$. So $a c \in \sqrt{I}$ or $b c \in \sqrt{I}$, a contradiction. Thus $a b I=0$. Similarly it can be easily verified that $b c I=a c I=0$.

(2) Suppose that $a i_{1} i_{2} \neq 0$ for some $i_{1}, i_{2} \in I$. Hence from (1) we have $a\left(b+i_{1}\right)\left(c+i_{2}\right)=a i_{1} i_{2} \neq 0$. It implies either $a\left(b+i_{1}\right) \in I$ or $a\left(c+i_{2}\right) \in \sqrt{I}$ or $\left(b+i_{1}\right)\left(c+i_{2}\right) \in \sqrt{I}$. Thus $a b \in I$ or $a c \in \sqrt{I}$ or $b c \in \sqrt{I}$, a contradiction. Therefore $a I^{2}=0$. Similarly, one can easily show that $b I^{2}=c I^{2}=0$.

Theorem 2.10. If $I$ is a weakly 2-absorbing primary ideal of $R$ that is not 2-absorbing primary, then $I^{3}=0$.

Proof. Suppose that $I$ is a weakly 2 -absorbing primary ideal that is not a 2 absorbing primary ideal of $R$. Then there exists $(a, b, c)$ a triple-zero of $I$ for some $a, b, c \in R$. Assume that $I^{3} \neq 0$. Hence $i_{1} i_{2} i_{3} \neq 0$ for some $i_{1}, i_{2}, i_{3} \in I$. By Theorem 2.9, we obtain $\left(a+i_{1}\right)\left(b+i_{2}\right)\left(c+i_{3}\right)=i_{1} i_{2} i_{3} \neq 0$. This implies that $\left(a+i_{1}\right)\left(b+i_{2}\right) \in I$ or $\left(a+i_{1}\right)\left(c+i_{3}\right) \in \sqrt{I}$ or $\left(b+i_{2}\right)\left(c+i_{3}\right) \in \sqrt{I}$. Thus we have $a b \in I$ or $a c \in \sqrt{I}$ or $b c \in \sqrt{I}$, a contradiction. Thus $I^{3}=0$.

Corollary 2.11. If $I$ is a weakly 2-absorbing primary ideal of $R$ that is not 2-absorbing primary, then $\sqrt{I}=\sqrt{0}$.

Recall that a ring $R$ is said to be reduced if $\sqrt{0}=0$.

Corollary 2.12. Let $R$ be a reduced ring and $I \neq 0$ be a proper ideal of $R$. Then $I$ is a weakly 2-absorbing primary ideal if and only if $I$ is a 2-absorbing primary ideal of $R$. 
The following example shows that a proper ideal $I$ of $R$ with the property $I^{3}=0$ need not be a weakly 2-absorbing primary ideal of $R$. We have the following example.

Example 2.13. Let $R=\mathbb{Z}_{90}$. Then $I=\{0,30,60\}$ is an ideal of $R$ and clearly $I^{3}=0$. Since $0 \neq 2 \cdot 3 \cdot 5=30 \in I, 2 \cdot 3=6 \notin I, 2 \cdot 5=10 \notin \sqrt{I}$, and $3 \cdot 5=15 \notin \sqrt{I}$, we conclude that $I$ is not a weakly 2 -absorbing primary ideal of $R$.

Let $I$ be a proper ideal of $R$. Since $\sqrt{\sqrt{I}}=\sqrt{I}$, we remind the reader again that $\sqrt{I}$ is a prime ideal of $R$ if and only if $\sqrt{I}$ is a primary ideal of $R$. We have the following result.

Theorem 2.14. Suppose that $\sqrt{0}$ is a prime (primary) ideal of $R$. Let $I$ be a proper ideal of $R$. Then $I$ is a weakly 2-absorbing primary ideal of $R$ if and only if $I$ is a 2-absorbing primary ideal of $R$.

Proof. Suppose that $I$ is a weakly 2-absorbing primary ideal of $R$. Assume that $a b c \in I$ for some $a, b, c \in R$. If $0 \neq a b c \in I$, then $a b \in I$ or $a c \in \sqrt{I}$ or $b c \in \sqrt{I}$. Hence assume that $a b c=0$ and $a b \notin I$. Since $a b c=0$ and $\sqrt{0}$ is a prime ideal of $R$, we conclude that $a \in \sqrt{0}$ or $b \in \sqrt{0}$ or $c \in \sqrt{0}$. Since $\sqrt{0} \subseteq \sqrt{I}$, we conclude that $a c \in \sqrt{0} \subseteq \sqrt{I}$ or $b c \in \sqrt{0} \subseteq \sqrt{I}$. Thus $I$ is a 2-absorbing primary ideal of $R$. The converse is clear.

Theorem 2.15. Suppose that $\{0\}$ has a triple-zero $(a, b, c)$ for some $a, b, c \in R$ such that $a b \notin \sqrt{0}$. Let $I$ be a weakly 2-absorbing primary ideal of $R$. Then $I$ is not a 2-absorbing primary ideal of $R$ if and only if $I \subseteq \sqrt{0}$.

Proof. Suppose that $I$ is not a 2-absorbing primary ideal of $R$. Then $I \subseteq \sqrt{0}$ by Corollary 2.11. Conversely, suppose that $I \subseteq \sqrt{0}$. By hypothesis, we conclude that $a b \notin I, a c \notin \sqrt{0}$, and $b c \notin \sqrt{0}$. Thus $(a, b, c)$ is a triple-zero of $I$. Hence $I$ is not a 2-absorbing primary ideal of $R$.

Theorem 2.16. Let $I_{1}, I_{2}, \ldots, I_{n}$ be weakly 2-absorbing primary ideals of $R$ such that every $I_{i}$ is not 2-absorbing primary. Then $I=\cap_{i=1}^{n} I_{i}$ is a weakly 2-absorbing primary ideal of $R$.

Proof. Observe that $\sqrt{I_{i}}=\sqrt{0}$ for each $1 \leq i \leq n$ by Corollary 2.11. Thus $\sqrt{I}=\sqrt{0}$. Suppose that $a, b, c \in R$ with $0 \neq a b c \in I$ and $a b \notin I$. Then $a b \notin I_{k}$ for some $1 \leq k \leq n$. Hence $b c \in \sqrt{I_{k}}=\sqrt{0}=\sqrt{I}$ or $a c \in \sqrt{I_{k}}=\sqrt{0}=\sqrt{I}$. Hence $I$ is a weakly 2-absorbing ideal of $R$.

Theorem 2.17. Let $f: R \rightarrow R^{\prime}$ be a homomorphism of commutative rings. Then the following statements hold.

(1) If $f$ is a monomorphism and $J^{\prime}$ is a weakly 2-absorbing primary ideal of $R^{\prime}$, then $f^{-1}\left(J^{\prime}\right)$ is a weakly 2-absorbing primary ideal of $R$.

(2) If $f$ is an epimorphism and $J$ is a weakly 2-absorbing primary ideal of $R$ containing $\operatorname{Ker}(f)$, then $f(J)$ is a weakly 2-absorbing primary ideal of $R^{\prime}$. 
Proof. (1) Let $a, b, c \in R$ such that $0 \neq a b c \in f^{-1}\left(J^{\prime}\right)$. Since $\operatorname{Ker}(f)=0$, we get $0 \neq f(a b c)=f(a) f(b) f(c) \in J^{\prime}$. Hence we have $f(a) f(b) \in J^{\prime}$ or $f(b) f(c) \in \sqrt{J^{\prime}}$ or $f(a) f(c) \in \sqrt{J^{\prime}}$, and thus $a b \in f^{-1}\left(J^{\prime}\right)$ or $b c \in f^{-1}\left(\sqrt{J^{\prime}}\right)$ or $a c \in f^{-1}\left(\sqrt{J^{\prime}}\right)$. Since $f^{-1}\left(\sqrt{J^{\prime}}\right)=\sqrt{f^{-1}\left(J^{\prime}\right)}$, we conclude that $f^{-1}\left(J^{\prime}\right)$ is a weakly 2-absorbing primary ideal of $R$.

(2) Let $a^{\prime}, b^{\prime}, c^{\prime} \in R^{\prime}$ and $0 \neq a^{\prime} b^{\prime} c^{\prime} \in f(J)$. Then there exist $a, b, c \in R$ such that $f(a)=a^{\prime}, f(b)=b^{\prime}, f(c)=c^{\prime}$ and $0 \neq f(a b c)=a^{\prime} b^{\prime} c^{\prime} \in f(J)$. Since $\operatorname{Ker}(f) \subseteq J$, we have $0 \neq a b c \in J$. It implies that $a b \in J$ or $a c \in \sqrt{J}$ or $b c \in \sqrt{J}$. It means that $a^{\prime} b^{\prime} \in f(J)$ or $a^{\prime} c^{\prime} \in f(\sqrt{J}) \subseteq \sqrt{f(J)}$ or $b^{\prime} c^{\prime} \in$ $f(\sqrt{J}) \subseteq \sqrt{f(J)}$. Thus $f(J)$ is a weakly 2-absorbing primary ideal of $R^{\prime}$.

Theorem 2.18. Let $I, J$ be proper ideals of $R$ with $I \subseteq J$. Then the followings statements hold.

(1) If $J$ is a weakly 2-absorbing primary ideal of $R$, then $J / I$ is a weakly 2-absorbing primary ideal of $R / I$.

(2) If $I$ is a 2-absorbing primary ideal of $R$ and $J / I$ is a weakly 2-absorbing primary ideal of $R / I$, then $J$ is a 2-absorbing primary ideal of $R$.

(3) If $I$ is a weakly 2-absorbing primary ideal of $R$ and $J / I$ is a weakly 2absorbing primary ideal of $R / I$, then $J$ is a weakly 2-absorbing primary ideal of $R$.

Proof. (1) It is obtained from Theorem 2.17.

(2) Let $a, b, c \in R$ and $a b c \in J$. If $a b c \in I$, then $a b \in I \subseteq J$ or $b c \in \sqrt{I} \subseteq \sqrt{J}$ or $a c \in \sqrt{I} \subseteq \sqrt{J}$. So we may assume that $a b c \notin I$. Then we have $I \neq$ $(a+I)(b+I)(c+I) \in J / I$. Since $J / I$ is a weakly 2-absorbing primary ideal of $R / I$, we conclude $(a+I)(b+I)=a b+I \in J / I$ or $(a+I)(c+I)=a c+I \in \sqrt{J / I}$ or $(b+I)(c+I)=b c+I \in \sqrt{J / I}$. It follows that $a b \in J$ or $a c \in \sqrt{J}$ or $b c \in \sqrt{J}$. Thus $J$ is a 2-absorbing primary ideal of $R$.

(3) Let $a, b, c \in R$ and $0 \neq a b c \in J$. Then by a similar argument as in (2), $J$ is a weakly 2 -absorbing primary ideal of $R$.

If $I, J$ are weakly 2-absorbing primary ideals of a ring $R$ such that $\sqrt{I}=\sqrt{J}$, then $I+J$ need not be a weakly 2 -absorbing primary ideal of $R$. We have the following example.

Example 2.19. Let $A=\mathbb{Z}_{2}[T, U, W, X, Y], H=\left(T^{2}, U^{2}, W X Y+T+\right.$ $U, T U, T W, T X, T Y, U W, U X, U Y) A$ be an ideal of $A$, and $R=A / H$. Then by construction of $R, I=(T A+H) / H=\{0, T+H\}$ and $J=(U A+H) / H=$ $\{0, U+H\}$ are weakly 2-absorbing primary ideals of $R$ such that $|I|=|J|=2$ and $\sqrt{I}=\sqrt{J}=\sqrt{0}($ in $\mathrm{R})=(T, U, W X Y) A / H$. Let $L=I+J=(H+$ $(T, U) A) / H$. Then $\sqrt{L}=\sqrt{0}$ (in $R$ ) and $L$ is not a weakly 2-absorbing primary ideal of $R$. For $0 \neq(W+H)(X+H)(Y+H)=W X Y+H=T+U+H \in L$, but $W X+H \notin L, W Y+H \notin \sqrt{L}$, and $X Y+H \notin \sqrt{L}$. 
For a commutative ring with $1 \neq 0$, let $Z(R)$ be the set of all zero-divisors of $R$.

Theorem 2.20. Let $S$ be a multiplicatively closed subset of $R$. Then

(1) If $I$ is a weakly 2-absorbing primary ideal of $R$ with $I \cap S=\varnothing$, then $S^{-1} I$ is a weakly 2-absorbing primary ideal of $S^{-1} R$.

(2) If $S^{-1} I$ is a weakly 2-absorbing primary ideal of $S^{-1} R$ such that $S \cap$ $Z_{I}(R)=\varnothing$ and $S \cap Z(R)=\varnothing$, then $I$ is a weakly 2-absorbing primary ideal of $R$.

Proof. (1) Let $a, b, c \in R, s, t, k \in S$ such that $0 \neq \frac{a}{s} \frac{b}{t} \frac{c}{k} \in S^{-1} I$. Then there exists $u \in S$ such that $0 \neq u a b c \in I$. Since $I$ is a weakly 2-absorbing primary ideal, we get either $u a b \in I$ or $b c \in \sqrt{I}$ or $u a c \in \sqrt{I}$. If $u a b \in I$, then $\frac{a}{s} \frac{b}{t}=\frac{u a b}{u s t} \in S^{-1} I$. If $b c \in \sqrt{I}$, then $\frac{b}{t} \frac{c}{k} \in S^{-1} \sqrt{I}=\sqrt{S^{-1} I}$. If $u a c \in \sqrt{I}$, then $\frac{a}{s} \frac{c}{k}=\frac{u a c}{u s k} \in \sqrt{S^{-1} I}$.

(2) Let $a, b, c \in R$ such that $0 \neq a b c \in I$. Since $S \cap Z(R)=\varnothing$, we have $0 \neq \frac{a b c}{1}=\frac{a}{1} \frac{b}{1} \frac{c}{1} \in S^{-1} I$. It follows either $\frac{a}{1} \frac{b}{1} \in S^{-1} I$ or $\frac{b}{1} \frac{c}{1} \in \sqrt{S^{-1} I}$ or $\frac{a}{1} \frac{c}{1} \in \sqrt{S^{-1} I}$. If $\frac{a}{1} \frac{b}{1}=\frac{a b}{1} \in S^{-1} I$, then $u a b \in I$ for some $u \in S$. Since $u \in S$ and $S \cap Z_{I}(R)=\varnothing$, we conclude $a b \in I$. If $\frac{b}{1} \frac{c}{1}=\frac{b c}{1} \in \sqrt{S^{-1} I}=S^{-1} \sqrt{I}$, then there exists $v \in S$ and a positive integer $n$ such that $(v b c)^{n}=v^{n} b^{n} c^{n} \in I$. Since $v \in S$, we have $v^{n} \notin Z_{I}(R)$. Thus $b^{n} c^{n} \in I$, and so $b c \in \sqrt{I}$. If $\frac{a}{1} \frac{c}{1} \in \sqrt{S^{-1} I}$, then similarly we obtain $a c \in \sqrt{I}$, and it completes the proof.

Theorem 2.21. Let $R_{1}$ and $R_{2}$ be commutative rings with $1 \neq 0, I$ be a proper ideal of $R_{1}$, and $R=R_{1} \times R_{2}$. Then the following statements are equivalent.

(1) $I \times R_{2}$ is a weakly 2-absorbing primary ideal of $R$.

(2) $I \times R_{2}$ is a 2-absorbing primary ideal of $R$.

(3) $I$ is a 2-absorbing primary ideal of $R_{1}$.

Proof. $(1) \Rightarrow(2)$ Since $I \times R_{2} \nsubseteq \sqrt{0}$, we conclude that $I \times R_{2}$ is a 2-absorbing primary ideal of $R$ by Corollary 2.11 .

$(2) \Rightarrow(3)$ Suppose that $I$ is not a 2 -absorbing primary ideal of $R_{1}$. Then there exist $a, b, c \in R_{1}$ such that $a b c \in I$, but $a b \notin I, b c \notin \sqrt{I}$, and $a c \notin \sqrt{I}$. Since $(a, 1)(b, 1)(c, 1) \in I \times R_{2}$, we have $(a, 1)(b, 1)=(a b, 1) \in I \times R_{2}$ or $(a, 1)(c, 1)=$ $(a c, 1) \in \sqrt{I \times R_{2}}=\sqrt{I} \times R_{2}$ or $(b, 1)(c, 1)=(b c, 1) \in \sqrt{I \times R_{2}}=\sqrt{I} \times R_{2}$. It follows that $a b \in I$ or $b c \in \sqrt{I}$ or $a c \in \sqrt{I}$, a contradiction. Thus $I$ is a 2-absorbing primary ideal of $R_{1}$.

$(3) \Rightarrow(1)$ Let $I$ be a 2-absorbing primary ideal of $R_{1}$. Then $I \times R_{2}$ is a 2 absorbing primary ideal of $R$ by [7, Theorem 2.23], and therefore (1) holds.

Theorem 2.22. Let $R_{1}$ and $R_{2}$ be commutative rings with $1 \neq 0, I_{1}, I_{2}$ be nonzero ideals of $R_{1}$ and $R_{2}$, respectively, and $R=R_{1} \times R_{2}$. If $I_{1} \times I_{2}$ is a proper ideal of $R$, then the following statements are equivalent.

(1) $I_{1} \times I_{2}$ is a weakly 2-absorbing primary ideal of $R$. 
(2) $I_{1}=R_{1}$ and $I_{2}$ is a 2-absorbing primary ideal of $R_{1}$ or $I_{2}=R_{2}$ and $I_{1}$ is a 2-absorbing primary ideal of $R_{1}$ or $I_{1}, I_{2}$ are primary ideals of $R_{1}, R_{2}$, respectively.

(3) $I_{1} \times I_{2}$ is a 2-absorbing primary ideal of $R$.

Proof. $(1) \Rightarrow(2)$ Assume that $I_{1} \times I_{2}$ is a weakly 2-absorbing primary ideal of $R$. If $I_{1}=R_{1}\left(I_{2}=R_{2}\right)$, then $I_{2}$ is a 2-absorbing primary ideal of $R_{2}\left(I_{1}\right.$ is a 2 -absorbing primary ideal of $R_{1}$ ) by Theorem 2.21. So we may assume that $I_{1} \neq R_{1}$ and $I_{2} \neq R_{2}$. Let $a, b \in R_{2}$ such that $a b \in I_{2}$ and let $0 \neq$ $x \in I_{1}$. Then $0 \neq(x, 1)(1, a)(1, b)=(x, a b) \in I_{1} \times I_{2}$. Since $I_{1}$ is proper, $(1, a)(1, b)=(1, a b) \notin \sqrt{I_{1} \times I_{2}}$. Hence we have $(x, 1)(1, a)=(x, a) \in I_{1} \times I_{2}$ or $(x, 1)(1, b)=(x, b) \in \sqrt{I_{1} \times I_{2}}$, and so $a \in I_{2}$ or $b \in \sqrt{I_{2}}$. Thus $I_{2}$ is a primary ideal of $R_{2}$. Similarly, it can be easily shown that $I_{1}$ is a primary ideal of $R_{1}$.

$(2) \Rightarrow(3)$ The proof is clear by [7, Theorem 2.23].

$(3) \Rightarrow(1)$ It is clear.

Theorem 2.23. Let $R_{1}$ and $R_{2}$ be commutative rings with $1 \neq 0$ and $R=$ $R_{1} \times R_{2}$. Then a nonzero proper ideal $I$ of $R$ is a weakly 2-absorbing primary ideal of $R$ that is not 2-absorbing primary if and only if one of the following conditions holds.

(1) $I=I_{1} \times I_{2}$, where $I_{1} \neq R_{1}$ is a nonzero weakly primary ideal of $R_{1}$ that is not primary and $I_{2}=0$ is a primary ideal of $R_{2}$.

(2) $I=I_{1} \times I_{2}$, where $I_{2} \neq R_{2}$ is a nonzero weakly primary ideal of $R_{2}$ that is not primary and $I_{1}=0$ is a primary ideal of $R_{1}$.

Proof. Suppose that $I$ is a nonzero weakly 2 -absorbing primary ideal of $R$ that is not 2-absorbing primary ideal. Then $I=I_{1} \times I_{2}$ for some ideals $I_{1}, I_{2}$ of $R_{1}$ and $R_{2}$, respectively. Assume that $I_{1} \neq 0$ and $I_{2} \neq 0$. Then $I$ is a 2-absorbing primary ideal of $R$ by Theorem 2.22, a contradiction. Therefore $I_{1}=0$ or $I_{2}=0$. Without loss of generality we may assume that $I_{2}=0$. We show that $I_{2}=0$ is a primary ideal of $R_{2}$. Let $a, b \in R_{2}$ such that $a b \in I_{2}$, and let $0 \neq x \in I_{1}$. Since $0 \neq(x, 1)(1, a)(1, b)=(x, a b) \in I$ and $(1, a b) \notin \sqrt{I}$, we obtain $(x, a)=(x, 1)(1, a) \in I$ or $(x, b)=(x, 1)(1, b) \in \sqrt{I}$, and so $a \in I_{2}$ or $b \in \sqrt{I_{2}}$. Thus $I_{2}=0$ is a primary ideal of $R_{2}$. Next, we show that $I_{1}$ is a weakly primary ideal of $R_{1}$. Observe that $I_{1} \neq R_{1}$. For if $I_{1}=R_{1}$, then $R_{1} \times 0$ is a 2-absorbing primary ideal of $R$ by [7, Theorem 2.23]. Let $0 \neq a b \in I_{1}$ for some $a, b \in R_{1}$. Since $0 \neq(a, 1)(b, 1)(1,0) \in I_{1} \times 0$ and $(a b, 1) \notin I_{1} \times 0$, we conclude $(a, 0)=(a, 1)(1,0) \in \sqrt{I_{1} \times 0}=\sqrt{I}$ or $(b, 0)=(b, 1)(1,0) \in \sqrt{I_{1} \times 0}=\sqrt{I}$. Thus $a \in I_{1}$ or $b \in \sqrt{I_{1}}$, and therefore $I_{1}$ is a weakly primary ideal of $R_{1}$. Now, we show that $I_{1}$ is not primary. Suppose that $I_{1}$ is a primary ideal of $R_{1}$. Since $I_{2}=\{0\}$ is a primary ideal of $R_{2}$, we conclude that $I=I_{1} \times I_{2}$ is a 2-absorbing primary ideal of $R$ by [7, Theorem 2.23], a contradiction. Thus $I_{1}$ is a weakly primary ideal of $R_{1}$ that is not primary.

Conversely, suppose that (1) holds. Assume that $(0,0) \neq\left(a, a^{\prime}\right)\left(b, b^{\prime}\right)\left(c, c^{\prime}\right) \in$ $I=I_{1} \times 0$. Since $a^{\prime} b^{\prime} c^{\prime}=0$ and $(0,0) \neq\left(a, a^{\prime}\right)\left(b, b^{\prime}\right)\left(c, c^{\prime}\right) \in I_{1} \times 0$, we conclude 
that $a b c \neq 0$. Assume $\left(a, a^{\prime}\right)\left(b, b^{\prime}\right) \notin I$. We consider three cases. Case one: Suppose that $a b \notin I_{1}$, but $a^{\prime} b^{\prime}=0$. Since $I_{1}$ is a weakly primary ideal of $R_{1}$, we have $c \in \sqrt{I_{1}}$. Since $I_{2}=0$ is a primary ideal of $R_{2}$, we have $a^{\prime}=0$ or $b^{\prime} \in \sqrt{I_{2}}$. Thus $\left(a, a^{\prime}\right)\left(c, c^{\prime}\right) \in \sqrt{I}$ or $\left(b, b^{\prime}\right)\left(c, c^{\prime}\right) \in \sqrt{I}$. Case two: Suppose that $a b \notin I_{1}$ and $a^{\prime} b^{\prime} \neq 0$. Then $\left(c, c^{\prime}\right) \in \sqrt{I_{1}} \times \sqrt{0}=\sqrt{I}$. Thus $\left(a, a^{\prime}\right)\left(c, c^{\prime}\right) \in \sqrt{I}$ or $\left(b, b^{\prime}\right)\left(c, c^{\prime}\right) \in \sqrt{I}$. Case three: Suppose that $a b \in I_{1}$, but $a^{\prime} b^{\prime} \neq 0$. Since $0 \neq a b \in I_{1}$ and $I_{1}$ is a weakly primary ideal of $R_{1}$, we have $a \in I_{1}$ or $b \in \sqrt{I_{1}}$. Since $a^{\prime} b^{\prime} \neq 0$ and $I_{2}=0$ is a primary ideal of $R_{2}$, we have $c^{\prime} \in \sqrt{I_{2}}$. Thus $\left(a, a^{\prime}\right)\left(c, c^{\prime}\right) \in \sqrt{I}$ or $\left(b, b^{\prime}\right)\left(c, c^{\prime}\right) \in \sqrt{I}$. Hence $I$ is a weakly 2 -absorbing primary ideal of $R$. Since $I_{1}$ is not a primary ideal of $R_{1}, I$ is not a 2-absorbing primary ideal of $R$ by [7, Theorem 2.23].

Theorem 2.24. Let $R=R_{1} \times R_{2} \times \cdots \times R_{n}$, where $2<n<\infty$, and $R_{1}, R_{2}$, $\ldots, R_{n}$ are commutative rings with $1 \neq 0$. Let $I$ be a nonzero proper ideal of $R$. Then the following statements are equivalent.

(1) $I$ is a weakly 2-absorbing primary ideal of $R$.

(2) $I$ is a 2-absorbing primary ideal of $R$.

(3) Either $I=\times_{j=1}^{n} I_{j}$ such that for some $k \in\{1, \ldots, n\}, I_{k}$ is a 2absorbing primary ideal of $R_{k}$, and $I_{j}=R_{j}$ for every $j \in\{1, \ldots, n\}-$ $\{k\}$, or $I=\times_{j=1}^{n} I_{j}$ such that for some $k, m \in\{1, \ldots, n\}, I_{k}$ is a primary ideal of $R_{k}, I_{m}$ is a primary ideal of $R_{m}$, and $I_{j}=R_{j}$ for every $j \in\{1, \ldots, n\}-\{k, m\}$.

Proof. $(1) \Leftrightarrow(2)$ Since $I$ is a proper ideal of $R$, we have $I=I_{1} \times \cdots \times I_{n}$, where every $I_{i}$ is an ideal of $R_{i}$, and $I_{j} \neq R_{j}$ for some $j \in\{1, \ldots, n\}$. Suppose that $I=I_{1} \times I_{2} \times \cdots \times I_{n} \neq 0$ is a weakly 2-absorbing primary ideal of $R$. Then there is an element $0 \neq\left(a_{1}, a_{2}, \ldots, a_{n}\right) \in I$. Hence $0 \neq$ $\left(a_{1}, a_{2}, \ldots, a_{n}\right)=\left(a_{1}, 1,1, \ldots, 1\right)\left(1, a_{2}, 1, \ldots, 1\right) \cdots\left(1,1, \ldots, a_{n}\right) \in I$ implies there is a $j \in\{1, \ldots, n\}$ such that $b_{j}=1$ and $\left(b_{1}, \ldots, b_{n}\right) \in \sqrt{I}=\sqrt{I_{1}} \times \cdots \times$ $\sqrt{I_{n}}$, where $b_{1}, \ldots, b_{n} \in\left\{1, a_{1}, \ldots, a_{n}\right\}$. Hence $\sqrt{I_{j}}=R_{j}$, and so $I_{j}=R_{j}$. Thus $\sqrt{I} \neq \sqrt{0}$, and hence by Corollary $2.11, I$ is a 2-absorbing primary ideal. The converse is obvious.

$(2) \Leftrightarrow(3)$ It is clear by [7, Theorem 2.24].

Theorem 2.25. Let $R_{1}, R_{2}$ and $R_{3}$ be commutative rings with $1 \neq 0$, and let $R=R_{1} \times R_{2} \times R_{3}$. Then every proper ideal of $R$ is a weakly 2-absorbing primary ideal of $R$ if and only if $R_{1}, R_{2}$, and $R_{3}$ are fields.

Proof. Suppose that every proper ideal of $R$ is a weakly 2-absorbing primary ideal of $R$. Without loss of generality, we may assume that $R_{1}$ is not a field. Then $R_{1}$ has a nonzero proper ideal $I$. Thus $J=I \times 0 \times 0$ is a weakly 2 -absorbing primary ideal of $R$, which is impossible by Theorem 2.24 .

Conversely, suppose that $R_{1}, R_{2}, R_{3}$ are fields. Then every nonzero proper ideal of $R$ is a 2 -absorbing ideal by [5, Theorem 3.4]. Since 0 is always weakly 2 -absorbing primary, the proof is completed. 
Theorem 2.26. Suppose that every proper ideal of $R$ is a weakly 2-absorbing primary ideal. Then $R$ has at most three incomparable (under inclusion) prime ideals.

Proof. Deny. Then there are $M_{1}, M_{2}, M_{3}$, and $M_{4}$ incomparable prime ideals of $R$. Let $I=M_{1} \cap M_{2} \cap M_{3}$. Hence $\sqrt{I}=\sqrt{M_{1}} \cap \sqrt{M_{2}} \cap \sqrt{M_{3}}$. Thus $\sqrt{I}$ is not a 2-absorbing ideal of $R$ by [3, Theorem 2.5]. So $I$ is not a 2-absorbing primary ideal of $R$ by [7, Theorem 2.2]. Hence $I^{3}=0$ by Theorem 2.10. Thus $I^{3}=M_{1}^{3} M_{2}^{3} M_{3}^{3}=0 \subseteq M_{4}$ implies that $M_{1} \subseteq M_{4}$ or $M_{2} \subseteq M_{4}$ or $M_{3} \subseteq M_{4}$, a contradiction. Thus $R$ has at most three incomparable (under inclusion) prime ideals.

In view of Theorem 2.26, we have the following result.

Corollary 2.27. Suppose that every proper ideal of $R$ is a weakly 2-absorbing primary ideal. Then $R$ has at most three maximal ideals.

Definition 2.28. Let $I$ be a weakly 2-absorbing primary ideal of $R$ and suppose that $I_{1} I_{2} I_{3} \subseteq I$ for some ideals $I_{1}, I_{2}$, and $I_{3}$ of $R$. We say $I$ is free triple-zero with respect to $I_{1} I_{2} I_{3}$ if $(a, b, c)$ is not a triple-zero of $I$ for every $a \in I_{1}, b \in I_{2}$, and $c \in I_{3}$.

Conjecture 1. Let I be a weakly 2-absorbing primary ideal of $R$ and suppose that $0 \neq I_{1} I_{2} I_{3} \subseteq I$ for some ideals $I_{1}, I_{2}$, and $I_{3}$ of $R$. Then $I$ is free triple-zero with respect to $I_{1} I_{2} I_{3}$.

Lemma 2.29. Let $I$ be a weakly 2-absorbing primary ideal of a ring $R$ and suppose that $a b J \subseteq I$ for some elements $a, b \in R$ and some ideal $J$ of $R$ such that $(a, b, c)$ is not a triple-zero of $I$ for every $c \in J$. If $a b \notin I$, then aJ $\subseteq \sqrt{I}$ or $b J \subseteq \sqrt{I}$.

Proof. Suppose that $a J \nsubseteq \sqrt{I}$ and $b J \nsubseteq \sqrt{I}$. Then $a j_{1} \notin \sqrt{I}$ and $b j_{2} \notin \sqrt{I}$ for some $j_{1}, j_{2} \in J$. Since $\left(a, b, j_{1}\right)$ is not a triple-zero of $I$ and $a b j_{1} \in I$ and $a b \notin I$ and $a j_{1} \notin \sqrt{I}$, we have $b j_{1} \in \sqrt{I}$. Since $\left(a, b, j_{2}\right)$ is not a triple-zero of $I$ and $a b j_{2} \in I$ and $a b \notin I$ and $b j_{2} \notin \sqrt{I}$, we have $a j_{2} \in \sqrt{I}$. Now, since $\left(a, b, j_{1}+j_{2}\right)$ is not a triple-zero of $I$ and $a b\left(j_{1}+j_{2}\right) \in I$ and $a b \notin I$, we have $a\left(j_{1}+j_{2}\right) \in \sqrt{I}$ or $b\left(j_{1}+j_{2}\right) \in \sqrt{I}$. Suppose that $a\left(j_{1}+j_{2}\right)=a j_{1}+a j_{2} \in \sqrt{I}$. Since $a j_{2} \in \sqrt{I}$, we have $a j_{1} \in \sqrt{I}$, a contradiction. Suppose that $b\left(j_{1}+j_{2}\right)=b j_{1}+b j_{2} \in \sqrt{I}$. Since $b j_{1} \in \sqrt{I}$, we have $b j_{2} \in \sqrt{I}$, a contradiction again. Thus $a J \subseteq \sqrt{I}$ or $b J \subseteq \sqrt{I}$.

Remark 1 . Let $I$ be a weakly 2 -absorbing primary ideal of $R$ and suppose that $I_{1} I_{2} I_{3} \subseteq I$ for some ideals $I_{1}, I_{2}$, and $I_{3}$ of $R$ such that $I$ is free triple-zero with respect to $I_{1} I_{2} I_{3}$. Then if $a \in I_{1}, b \in I_{2}$, and $c \in I_{3}$, then $a b \in I$ or $a c \in \sqrt{I}$ or $b c \in \sqrt{I}$.

Let $I$ be a weakly 2 -absorbing primary ideal of $R$. In view of the below result, one can see that Conjecture 1 is valid if and only if whenever $0 \neq I_{1} I_{2} I_{3} \subseteq I$ for some ideals $I_{1}, I_{2}, I_{3}$ of $R$, then $I_{1} I_{2} \subseteq I$ or $I_{2} I_{3} \subseteq \sqrt{I}$ or $I_{1} I_{3} \subseteq \sqrt{I}$. 
Theorem 2.30. Let $I$ be a weakly 2-absorbing primary ideal of $R$ and suppose that $0 \neq I_{1} I_{2} I_{3} \subseteq I$ for some ideals $I_{1}, I_{2}, I_{3}$ of $R$ such that $I$ is free triple-zero with respect to $I_{1} I_{2} I_{3}$. Then $I_{1} I_{2} \subseteq I$ or $I_{2} I_{3} \subseteq \sqrt{I}$ or $I_{1} I_{3} \subseteq \sqrt{I}$.

Proof. Suppose that $I$ is a weakly 2 -absorbing primary ideal of $R$ and $0 \neq$ $I_{1} I_{2} I_{3} \subseteq I$ for some ideals $I_{1}, I_{2}, I_{3}$ of $R$ such that $I$ is free triple-zero with respect to $I_{1} I_{2} I_{3}$. Suppose that $I_{1} I_{2} \nsubseteq I I$. By Remark 1, we proceed with the same argument as in the proof of [7, Theorem 2.19]. We show that $I_{1} I_{3} \subseteq \sqrt{I}$ or $I_{2} I_{3} \subseteq \sqrt{I}$. Suppose that neither $I_{1} I_{3} \subseteq \sqrt{I}$ nor $I_{2} I_{3} \subseteq \sqrt{I}$. Then there are $q_{1} \in I_{1}$ and $q_{2} \in I_{2}$ such that neither $q_{1} I_{3} \subseteq \sqrt{I}$ nor $q_{2} I_{3} \subseteq \sqrt{I}$. Since $q_{1} q_{2} I_{3} \subseteq I$ and neither $q_{1} I_{3} \subseteq \sqrt{I}$ nor $q_{2} I_{3} \subseteq \sqrt{I}$, we have $q_{1} q_{2} \in I$ by Lemma 2.29 .

Since $I_{1} I_{2} \nsubseteq I$, we have $a b \notin I$ for some $a \in I_{1}, b \in I_{2}$. Since $a b I_{3} \subseteq I$ and $a b \notin I$, we have $a I_{3} \subseteq \sqrt{I}$ or $b I_{3} \subseteq \sqrt{I}$ by Lemma 2.29. We consider three cases. Case one: Suppose that $a \bar{I}_{3} \subseteq \sqrt{I}$, but $b I_{3} \nsubseteq \sqrt{I}$. Since $q_{1} b I_{3} \subseteq I$ and neither $b I_{3} \subseteq \sqrt{I}$ nor $q_{1} I_{3} \subseteq \sqrt{I}$, we conclude that $q_{1} b \in I$ by Lemma 2.29. Since $\left(a+q_{1}\right) b I_{3} \subseteq I$ and $a I_{3} \subseteq \sqrt{I}$, but $q_{1} I_{3} \nsubseteq \sqrt{I}$, we conclude that $\left(a+q_{1}\right) I_{3} \nsubseteq \sqrt{I}$. Since neither $b I_{3} \subseteq \sqrt{I}$ nor $\left(a+q_{1}\right) I_{3} \subseteq \sqrt{I}$, we conclude that $\left(a+q_{1}\right) b \in I$ by Lemma 2.29. Since $\left(a+q_{1}\right) b=a b+q_{1} b \in I$ and $q_{1} b \in I$, we conclude that $a b \in I$, a contradiction. Case two: Suppose that $b I_{3} \subseteq \sqrt{I}$, but $a I_{3} \nsubseteq \sqrt{I}$. Since $a q_{2} I_{3} \subseteq I$ and neither $a I_{3} \subseteq \sqrt{I}$ nor $q_{2} I_{3} \subseteq \sqrt{I}$, we conclude that $a q_{2} \in I$. Since $a\left(b+q_{2}\right) I_{3} \subseteq I$ and $b I_{3} \subseteq \sqrt{I}$, but $q_{2} I_{3} \nsubseteq \sqrt{I}$, we conclude that $\left(b+q_{2}\right) I_{3} \nsubseteq \sqrt{I}$. Since neither $a I_{3} \subseteq \sqrt{I}$ nor $\left(b+q_{2}\right) I_{3} \subseteq \sqrt{I}$, we conclude that $a\left(b+q_{2}\right) \in I$ by Lemma 2.29. Since $a\left(b+q_{2}\right)=a b+a q_{2} \in I$ and $a q_{2} \in I$, we conclude that $a b \in I$, a contradiction. Case three: Suppose that $a I_{3} \subseteq \sqrt{I}$ and $b I_{3} \subseteq \sqrt{I}$. Since $b I_{3} \subseteq \sqrt{I}$ and $q_{2} I_{3} \nsubseteq \sqrt{I}$, we conclude that $\left(b+q_{2}\right) I_{3} \nsubseteq \sqrt{I}$. Since $q_{1}\left(b+q_{2}\right) I_{3} \subseteq I$ and neither $q_{1} I_{3} \subseteq \sqrt{I}$ nor $\left(b+q_{2}\right) I_{3} \subseteq \sqrt{I}$, we conclude that $q_{1}\left(b+q_{2}\right)=q_{1} b+q_{1} q_{2} \in I$ by Lemma 2.29. Since $q_{1} q_{2} \in I$ and $q_{1} b+q_{1} q_{2} \in I$, we conclude that $b q_{1} \in I$. Since $a I_{3} \subseteq \sqrt{I}$ and $q_{1} I_{3} \nsubseteq \sqrt{I}$, we conclude that $\left(a+q_{1}\right) I_{3} \nsubseteq \sqrt{I}$. Since $\left(a+q_{1}\right) q_{2} I_{3} \subseteq I$ and neither $q_{2} I_{3} \subseteq \sqrt{I}$ nor $\left(a+q_{1}\right) I_{3} \subseteq \sqrt{I}$, we conclude that $\left(a+q_{1}\right) q_{2}=a q_{2}+q_{1} q_{2} \in I$ by Lemma 2.29. Since $q_{1} q_{2} \in I$ and $a q_{2}+q_{1} q_{2} \in I$, we conclude that $a q_{2} \in I$. Now, since $\left(a+q_{1}\right)\left(b+q_{2}\right) I_{3} \subseteq I$ and neither $\left(a+q_{1}\right) I_{3} \subseteq \sqrt{I}$ nor $\left(b+q_{2}\right) I_{3} \subseteq \sqrt{I}$, we conclude that $\left(a+q_{1}\right)\left(b+q_{2}\right)=a b+a q_{2}+b q_{1}+q_{1} q_{2} \in I$ by Lemma 2.29 . Since $a q_{2}, b q_{1}, q_{1} q_{2} \in I$, we have $a q_{2}+b q_{1}+q_{1} q_{2} \in I$. Since $a b+a q_{2}+b q_{1}+q_{1} q_{2} \in I$ and $a q_{2}+b q_{1}+q_{1} q_{2} \in I$, we conclude that $a b \in I$, a contradiction. Hence $I_{1} I_{3} \subseteq \sqrt{I}$ or $I_{2} I_{3} \subseteq \sqrt{I}$.

\section{A visit to weakly prime ideals and weakly 2-absorbing ideals}

Definition 3.1. Let $I$ be a weakly prime ideal of $R$. We say $(a, b)$ is a twin-zero of $I$ if $a b=0, a \notin I$, and $b \notin I$.

In this section, we use the concept "twin-zero"in order to give alternative proofs to some results in [2]. 
Note that if $I$ is a weakly prime ideal of $R$ that is not a prime ideal, then $I$ has a twin-zero $(a, b)$ for some $a, b \in R$.

Theorem 3.2. Let $I$ be a weakly prime ideal of $R$ and suppose that $(a, b)$ is a twin-zero of $I$ for some $a, b \in R$. Then $a I=b I=0$.

Proof. Suppose that $a I \neq 0$. Then there exists $i \in I$ such that $a i \neq 0$. Hence $a(b+i) \neq 0$. Since $a \notin I$ and $I$ is weakly prime, we have $b+i \in I$, and hence $b \in I$, a contradiction. Thus $a I=0$. Similarly, it can be easily verified that $b I=0$.

Theorem 3.3 ([2, Theorem 1]). Let I be a weakly prime ideal of $R$. If $I$ is not prime, then $I^{2}=0$.

Proof. Let $(a, b)$ be a twin-zero of $I$. Suppose that $i_{1} i_{2} \neq 0$ for some $i_{1}, i_{2} \in I$. Then by Theorem 3.2 , we have $\left(a+i_{1}\right)\left(b+i_{2}\right)=i_{1} i_{2} \neq 0$. Thus $\left(a+i_{1}\right) \in I$ or $\left(b+i_{2}\right) \in I$, and hence $a \in I$ or $b \in I$, a contradiction. Therefore $I^{2}=0$.

Theorem 3.4 ([2, Theorem 4]). Let I be a weakly prime ideal of $R$. If $I$ is not prime, then $I \subseteq \sqrt{0}$ and $I \sqrt{0}=0$.

Proof. Suppose that $I$ is not prime. Then $I \subseteq \sqrt{0}$ by Theorem 3.3. Let $w \in \sqrt{0}$. If $w \in I$, then $w I=0$ by Theorem 3.3. Thus assume that $w \notin I$ and $w I \neq 0$. Hence $w i \neq 0$ for some $i \in I$. Let $m$ be the least positive integer such that $w^{m}=0$. Since $w\left(w^{m-1}+i\right)=w i \neq 0$ and $w \notin I$, we have $w^{m-1}+i \in I$, and hence $w^{m-1} \in I$. Since $0 \neq w^{m-1} \in I$ and $I$ is weakly prime, we conclude that $w \in I$, a contradiction. Thus $w I=0$. Hence $I \sqrt{0}=0$.

Theorem 3.5. Let $I$ be a weakly prime ideal of $R$ and suppose that $(a, b)$ is a twin-zero of $I$. If ar $\in I$ for some $r \in R$, then $a r=0$.

Proof. Suppose that $0 \neq a r \in I$ for some $r \in R$. Then $r \in I$. Thus $a r=0$ by Theorem 3.2, a contradiction.

Theorem 3.6. Let $I$ be a weakly prime ideal of $R$ and suppose that $A B \subseteq I$ for some ideals $A, B$ of $R$. If I has a twin-zero $(a, b)$ for some $a \in A$ and $b \in B$, then $A B=0$.

Proof. Suppose that $I$ has a twin-zero $(a, b)$ for some $a \in A$ and $b \in B$ and assume that $c d \neq 0$ for some $c \in A$ and $d \in B$. Then $c \in I$ or $d \in I$. Without loss of generality, we may assume that $c \in I$. Since $I^{2}=0$ by Theorem 3.2 and $0 \neq c d \in I$, we conclude that $d \notin I$. Since $a d \in I$, we have $a d=0$ by Theorem 3.5. Since $(a+c) d=c d \neq 0$ and $d \notin I$, we have $a+c \in I$. Hence $a \in I$, a contradiction. Thus $A B=0$.

Theorem 3.7 ([2, Theorem 3(4)]). Let I be a weakly prime ideal of $R$ and suppose that $0 \neq A B \subseteq I$ for some ideals $A, B$ of $R$. Then $A \subseteq I$ or $B \subseteq I$. 
Proof. Since $0 \neq A B \subseteq I$, we conclude that for every $a \in A$ and $b \in B$, we have $a \in I$ or $b \in I$ by Theorem 3.6. Without loss of generality, assume that $B \nsubseteq I$. Hence $b \notin I$ for some $b \in B$. Let $a \in A$. Since $a b \in I$ and $b \notin I$, we have $a \in I$. Thus $A \subseteq I$.

We recall the following definition from [6].

Definition 3.8. Let $I$ be a weakly 2-absorbing ideal of a $\operatorname{ring} R$ and $a, b, c \in R$. We say $(a, b, c)$ is a triple-zero of $I$ if $a b c=0, a b \notin I, b c \notin I$, and $a c \notin I$.

Definition 3.9. Let $I$ be a weakly 2-absorbing ideal of $R$ and suppose that $I_{1} I_{2} I_{3} \subseteq I$ for some ideals $I_{1}, I_{2}$, and $I_{3}$ of $R$. We say $I$ is free triple-zero with respect to $I_{1} I_{2} I_{3}$ if $(a, b, c)$ is not a triple-zero of $I$ for every $a \in I_{1}, b \in I_{2}$, and $c \in I_{3}$.

Conjecture 2. Let $I$ be a weakly 2-absorbing ideal of $R$ and suppose that $0 \neq I_{1} I_{2} I_{3} \subseteq I$ for some ideals $I_{1}, I_{2}$, and $I_{3}$ of $R$. Then $I$ is free triple-zero with respect to $I_{1} I_{2} I_{3}$.

Lemma 3.10. Let $I$ be a weakly 2-absorbing ideal of a ring $R$ and suppose that $a b J \subseteq I$ for some elements $a, b \in R$ and some ideal $J$ of $R$ such that $(a, b, c)$ is not a triple-zero of I for every $c \in J$. If $a b \notin I$, then $a J \subseteq I$ or $b J \subseteq I$.

Proof. Suppose that $a J \nsubseteq I$ and $b J \nsubseteq I$. Then $a j_{1} \notin I$ and $b j_{2} \notin I$ for some $j_{1}, j_{2} \in J$. Since $\left(a, b, j_{1}\right)$ is not a triple-zero of $I$ and $a b j_{1} \in I$ and $a b \notin I$ and $a j_{1} \notin I$, we have $b j_{1} \in I$. Since $\left(a, b, j_{2}\right)$ is not a triple-zero of $I$ and $a b j_{2} \in I$ and $a b \notin I$ and $b j_{2} \notin I$, we have $a j_{2} \in I$. Now, since $\left(a, b, j_{1}+j_{2}\right)$ is not a triple-zero of $I$ and $a b\left(j_{1}+j_{2}\right) \in I$ and $a b \notin I$, we have $a\left(j_{1}+j_{2}\right) \in I$ or $b\left(j_{1}+j_{2}\right) \in I$. Suppose that $a\left(j_{1}+j_{2}\right)=a j_{1}+a j_{2} \in I$. Since $a j_{2} \in I$, we have $a j_{1} \in I$, a contradiction. Suppose that $b\left(j_{1}+j_{2}\right)=b j_{1}+b j_{2} \in I$. Since $b j_{1} \in I$, we have $b j_{2} \in I$, a contradiction again. Thus $a J \subseteq I$ or $b J \subseteq I$.

Remark 2. Let $I$ be a weakly 2-absorbing ideal of $R$ and suppose that $I_{1} I_{2} I_{3} \subseteq I$ for some ideals $I_{1}, I_{2}$, and $I_{3}$ of $R$ such that $I$ is free triple-zero with respect to $I_{1} I_{2} I_{3}$. Then if $a \in I_{1}, b \in I_{2}$, and $c \in I_{3}$, then $a b \in I$ or $a c \in I$ or $b c \in I$.

Let $I$ be a weakly 2-absorbing ideal of $R$. In view of the below result, one can see that Conjecture 2 is valid if and only if whenever $0 \neq I_{1} I_{2} I_{3} \subseteq I$ for some ideals $I_{1}, I_{2}, I_{3}$ of $R$, then $I_{1} I_{2} \subseteq I$ or $I_{2} I_{3} \subseteq I$ or $I_{1} I_{3} \subseteq I$.

Theorem 3.11. Let $I$ be a weakly 2-absorbing ideal of $R$ and suppose that $0 \neq I_{1} I_{2} I_{3} \subseteq I$ for some ideals $I_{1}, I_{2}, I_{3}$ of $R$ such that $I$ is free triple-zero with respect to $I_{1} I_{2} I_{3}$. Then $I_{1} I_{2} \subseteq I$ or $I_{2} I_{3} \subseteq I$ or $I_{1} I_{3} \subseteq I$.

Proof. Suppose that $I$ is a weakly 2-absorbing ideal of $R$ and $0 \neq I_{1} I_{2} I_{3} \subseteq I$ for some ideals $I_{1}, I_{2}, I_{3}$ of $R$ such that such that $I$ is free triple-zero with respect to $I_{1} I_{2} I_{3}$. Suppose that $I_{1} I_{2} \nsubseteq I$. By Remark 2, we proceed with a similar argument as in the proof of [7, Theorem 2.19]. We show that $I_{1} I_{3} \subseteq I$ or $I_{2} I_{3} \subseteq I$. Suppose that neither $I_{1} I_{3} \subseteq I$ nor $I_{2} I_{3} \subseteq I$. Then there are 
$q_{1} \in I_{1}$ and $q_{2} \in I_{2}$ such that neither $q_{1} I_{3} \subseteq I$ nor $q_{2} I_{3} \subseteq I$. Since $q_{1} q_{2} I_{3} \subseteq I$ and neither $q_{1} I_{3} \subseteq I$ nor $q_{2} I_{3} \subseteq I$, we have $q_{1} q_{2} \in I$ by Lemma 3.10.

Since $I_{1} I_{2} \nsubseteq I$, we have $a b \notin I$ for some $a \in I_{1}, b \in I_{2}$. Since $a b I_{3} \subseteq I$ and $a b \notin I$, we have $a I_{3} \subseteq I$ or $b I_{3} \subseteq I$ by Lemma 3.10. We consider three cases. Case one: Suppose that $a I_{3} \subseteq I$, but $b I_{3} \nsubseteq I$. Since $q_{1} b I_{3} \subseteq I$ and neither $b I_{3} \subseteq I$ nor $q_{1} I_{3} \subseteq I$, we conclude that $q_{1} b \in I$ by Lemma 3.10. Since $\left(a+q_{1}\right) b I_{3} \subseteq I$ and $a I_{3} \subseteq I$, but $q_{1} I_{3} \nsubseteq I$, we conclude that $\left(a+q_{1}\right) I_{3} \nsubseteq I$. Since neither $b I_{3} \subseteq I$ nor $\left(a+q_{1}\right) I_{3} \subseteq I$, we conclude that $\left(a+q_{1}\right) b \in I$ by Lemma 3.10. Since $\left(a+q_{1}\right) b=a b+q_{1} b \in I$ and $q_{1} b \in I$, we conclude that $a b \in I$, a contradiction. Case two: Suppose that $b I_{3} \subseteq I$, but $a I_{3} \nsubseteq I$. Since $a q_{2} I_{3} \subseteq I$ and neither $a I_{3} \subseteq I$ nor $q_{2} I_{3} \subseteq I$, we conclude that $a q_{2} \in I$. Since $a\left(b+q_{2}\right) I_{3} \subseteq$ $I$ and $b I_{3} \subseteq I$, but $q_{2} I_{3} \nsubseteq I$, we conclude that $\left(b+q_{2}\right) I_{3} \nsubseteq I$. Since neither $a I_{3} \subseteq I$ nor $\left(b+q_{2}\right) I_{3} \subseteq I$, we conclude that $a\left(b+q_{2}\right) \in I$ by Lemma 3.10. Since $a\left(b+q_{2}\right)=a b+a q_{2} \in I$ and $a q_{2} \in I$, we conclude that $a b \in I$, a contradiction. Case three: Suppose that $a I_{3} \subseteq I$ and $b I_{3} \subseteq I$. Since $b I_{3} \subseteq I$ and $q_{2} I_{3} \nsubseteq I$, we conclude that $\left(b+q_{2}\right) I_{3} \nsubseteq I$. Since $q_{1}\left(b+q_{2}\right) I_{3} \subseteq I$ and neither $q_{1} I_{3} \subseteq I$ nor $\left(b+q_{2}\right) I_{3} \subseteq I$, we conclude that $q_{1}\left(b+q_{2}\right)=q_{1} b+q_{1} q_{2} \in I$ by Lemma 3.10. Since $q_{1} q_{2} \in I$ and $q_{1} b+q_{1} q_{2} \in I$, we conclude that $b q_{1} \in I$. Since $a I_{3} \subseteq I$ and $q_{1} I_{3} \nsubseteq I$, we conclude that $\left(a+q_{1}\right) I_{3} \nsubseteq I$. Since $\left(a+q_{1}\right) q_{2} I_{3} \subseteq I$ and neither $q_{2} I_{3} \subseteq I$ nor $\left(a+q_{1}\right) I_{3} \subseteq I$, we conclude that $\left(a+q_{1}\right) q_{2}=a q_{2}+q_{1} q_{2} \in I$ by Lemma 3.10. Since $q_{1} q_{2} \in I$ and $a q_{2}+q_{1} q_{2} \in I$, we conclude that $a q_{2} \in I$. Now, since $\left(a+q_{1}\right)\left(b+q_{2}\right) I_{3} \subseteq I$ and neither $\left(a+q_{1}\right) I_{3} \subseteq I$ nor $\left(b+q_{2}\right) I_{3} \subseteq I$, we conclude that $\left(a+q_{1}\right)\left(b+q_{2}\right)=a b+a q_{2}+b q_{1}+q_{1} q_{2} \in I$ by Lemma 3.10. Since $a q_{2}, b q_{1}, q_{1} q_{2} \in I$, we have $a q_{2}+b q_{1}+q_{1} q_{2} \in I$. Since $a b+a q_{2}+b q_{1}+q_{1} q_{2} \in I$ and $a q_{2}+b q_{1}+q_{1} q_{2} \in I$, we conclude that $a b \in I$, a contradiction. Hence $I_{1} I_{3} \subseteq I$ or $I_{2} I_{3} \subseteq I$.

Acknowledgement. We would like to thank the referee for his/her great efforts in proofreading the manuscript and for the thoughtful comments. The second-named author and the third-named author were supported by the Scientific Research Project Program of Marmara University (BAPKO).

\section{References}

[1] D. D. Anderson and M. Bataineh, Generalizations of prime ideals, Comm. Algebra 36 (2008), no. 2, 686-696.

[2] D. D. Anderson and E. Smith, Weakly prime ideals, Houston J. Math. 29 (2003), no. 4, $831-840$.

[3] D. F. Anderson and A. Badawi, On n-absorbing ideals of commutative rings, Comm. Algebra 39 (2011), no. 5, 1646-1672.

[4] S. Ebrahimi Atani and F. Farzalipour, On weakly primary ideals, Georgian Math. J. 12 (2005), no. 3, 423-429.

[5] A. Badawi, On 2-absorbing ideals of commutative rings, Bull. Austral. Math. Soc. 75 (2007), no. 3, 417-429.

[6] A. Badawi and A. Y. Darani, On weakly 2-absorbing ideals of commutative rings, Houston J. Math. 39 (2013), no. 2, 441-452. 
[7] A. Badawi, U. Tekir, and E. Yetkin, On 2-absorbing primary ideals in commutative rings, Bull. Korean Math. Soc. (in press)

[8] A. Y. Darani and E. R. Puczylowski, On 2-absorbing commutative semigroups and their applications to rings, Semigroup Forum 86 (2013), no. 1, 83-91.

[9] M. Ebrahimpour and R. Nekooei, On generalizations of prime ideals, Comm. Algebra 40 (2012), no. 4, 1268-1279.

[10] R. Gilmer, Multiplicative Ideal Theory, Queen's Papers Pure Appl. Math. 90, Queen's University, Kingston, 1992.

[11] J. Huckaba, Rings with Zero-Divisors, New York/Basil: Marcel Dekker, 1988.

[12] S. Payrovi and S. Babaei, On the 2-absorbing ideals, Int. Math. Forum 7 (2012), no. 5-8, 265-271.

AYMAN BADAWI

Department of Mathematics \& Statistics

American University of Sharjah

P.O.Box 26666, Sharjah, United Arab Emirates

E-mail address: abadawi@aus.edu

UNSAL TEKIR

Department of Mathematics

MARMARA UNIVERSITY

ISTANBUL, TURKEY

E-mail address: utekir@marmara.edu.tr

ECE YeTKIN

Department of Mathematics

MARMARA UNIVERSITY

ISTANBUL, TURKEY

E-mail address: ece.yetkin@marmara.edu.tr 